

Alexandre EVIN-LECLERC

Consultant secteur public

\title{
Dématérialisation et digitalisation de la fonction finance : enjeux et opportunités pour le bloc local
}

Mołs-clés: gestion publique - collectivités territoriales - dématérialisation - facturation électronique

\section{La dématérialisation des comptes, des relations avec le comptable public,} des factures et des marchés, modifie considérablement l'exercice de la fonction financière comme le montrent les témoignages réunis par le Forum pour la gestion des villes et des collectivités territoriales.

e Forum pour la Gestion des Villes et des Collectivités Territoriales et la Revue Gestion \& Finances Publiques, organisent un cycle de formations-actions dédié aux cadres dirigeants des collectivités territoriales : les rendez-vous de la transformation de la fonction finance.

Jeudi 16 mars 2017, s'est tenu le premier rendezvous «Dématérialisation et digitalisation de la fonction finance : enjeux et opportunités pour le bloc local ». Y participaient: Sylvie BRENNER, Chef de la mission de déploiement de la dématérialisation à la DGFiP, Geneviève OLLIER, Comptable du Trésor, Anne-Marie DUBOST, Directrice des finances de la ville de Vincennes, Pascale PRADELS, Directrice financière et comptable de l'UGAP.

\section{Alexandre EVIN-LECLERC, Consultant secteur public}

Alexandre Evin-Leclerc, qui animait la rencontre, a introduit les débats en rappelant plusieurs événements significatifs qui contribuent à modifier en profondeur la fonction finance au sein du bloc local :
$\mathrm{EPCl}^{1}$ et régions au périmètre élargi, création de métropoles, évolution des modes de financement, ouverture des données publiques ou encore, lancement de la phase expérimentale de certification des comptes locaux...

La digitalisation des comptes publics locaux s'inscrit ainsi dans un continuum de réformes déjà initiées. Que ce soit la généralisation de la facture électronique, l'enrichissement de l'offre aux citoyens utilisateurs, tout concourt à un service public plus performant, moins chronophage, plus écoresponsable et surtout, plus proche du citoyen.

Dès lors, comment identifier les facteurs clés de réussite de la dématérialisation, quels en sont les avantages et la valeur ajoutée, comment caractériser les prochains enjeux de ces démarches et comment les accompagner? La dématérialisation ne se résume pas simplement à une mise en conformité réglementaire ou à un chantier système d'information. II s'agit également (et surtout) d'une démarche managériale, organisationnelle et humaine.
1 Établissement public de coopération intercommunale. 
Sylvie BRENNER,

Chef de la mission de déploiement de la dématérialisation à la DGFiP2

Première intervenante, Sylvie BRENNER a tenté de répondre à la question: " en quoi l'offre de services dématérialisés contribue-t-elle à améliorer le service public?»

1. La dématérialisation des échanges ordonnateurscomptables a été rendue possible par le changement de protocole informatique (obligation réglementaire d'adoption du PES ${ }^{3}$ V2 au 1er janvier 2015) mais aussi par la concertation menée avec différents partenaires, dans le cadre de la structure nationale partenariale.

La dématérialisation permet ainsi de créer une administration numérique de référence (développement des moyens modernes de paiement, dématérialisation des procédures fiscales et des actes de poursuites...), mais aussi d'optimiser des processus de travail et de générer ainsi de nombreux gains (qualitatifs, financiers, d'image, de temps, de traçabilité, de sécurité...).

2. Avec les échéances imposées, comme la disparition complète du papier, au 1er janvier 2017 pour les métropoles et au 1 $1^{\text {er }}$ janvier 2019 pour les communes et $\mathrm{EPCl}$ de plus de 10000 habitants, la question de la dématérialisation demande une véritable réflexion et une démarche pour les collectivités. Elle doit devenir une préoccupation politique, afin de devenir un projet partagé.

Par exemple, le déploiement de la solution Chorus Pro dans les entités publiques implique de nombreuses actions:

- préparation au lancement : établir et suivre un planning de travail ;

- organisation : analyser les impacts de la facturation électronique sur l'organisation, définir et mettre en œuvre les évolutions nécessaires ;

- mise à niveau des systèmes d'informations : garantir la faisabilité technique des projets de dématérialisation au regard des systèmes d'informations, en particulier le raccordement à la solution Chorus Pro ;

- communication : sensibiliser et faire adhérer les acteurs internes et externes à la démarche de dématérialisation ;

- formation : former les utilisateurs en fonction de leurs besoins et au plus près de la phase de démarrage ;

- accompagnement au changement : préparer et mettre en œuvre les actions permettant le démarrage de la solution mutualisée Chorus Pro dans les meilleures conditions ;

- démarrage : sécuriser la phase de démarrage et assurer la transition vers le fonctionnement permanent.

La démarche des collectivités doit donc s'appuyer sur la construction d'un flux de travail qui doit permettre d'embarquer l'ensemble du processus, de la commande à la livraison du bien, jusqu'à la transmission comptable. Cette mise en œuvre est indispensable à un projet de dématérialisation structuré.

3. Après le PES V2, la DGFiP travaille activement sur différents projets à destination des organismes publics locaux :

- PES PJ : il permet la dématérialisation des pièces justificatives et leur transmission de I'ordonnateur au comptable ;

- PES ASAP (en cours de déploiement depuis fin 2016) : il permet la gestion des Avis des Sommes À Payer ;

- PES Retour : structuré par domaine fonctionnel (PES Retour Dépense et PES Retour Recette), il permet d'informer l'ordonnateur des actions menées par le comptable ;

- ATLAS et ORC ${ }^{4}$ (actuellement testés en phase de préfiguration par une vingtaine de collectivités pilote avant généralisation) : un audit scientifique et technique réalisé par le SIAF5 a validé la capacité silo ATLAS à assurer l'archivage intermédiaire des pièces justificatives et comptables des collectivités locales sur 10 ans.

- ENSU6 (prévu pour 2018) : il permettra la mise en ligne de l'ensemble des créances de l'usager recouvrées par les services de la DGFiP ;

- données essentielles pour le recensement des marchés publics (prévu pour 2018) : définir et mettre en place un schéma unique permettant de satisfaire trois objectifs : le recensement des marchés, la publication des données essentielles (open data), l'alimentation des données marché pour le comptable public.

4. Les perspectives de la dématérialisation ne s'arrêtent pas là. Elle permettra, à terme, de créer de nouveaux processus métier et de nouvelles pratiques comptables.

"La donnée est une mine à partager. II n'y a que des bénéfices à en tirer pour les usagers et les services publics. »

Quelques exemples peuvent être signalés.

Le contrôle allégé en partenariat permet un contrôle a posteriori, un allègement dans la transmission des pièces justificatives après réalisation d'un audit conjoint ordonnateur, comptable de la chaîne de la dépense ayant permis d'obtenir l'assurance d'une fiabilisation des opérations concernées.

La mutualisation d'un certain nombre de contrôles ordonnateur-comptable dans le cadre d'une équipe mixte, service facturier, placée, sous l'autorité du comptable, est expérimentée et autorise des gains d'efficacité, tout en fiabilisant le processus de réception et traitement des factures.

La Ville de Lyon utilise, depuis juin 2016, la plateforme France Connect. Le calcul automatique des 
droits à prestations soumises au quotient familial évite aux usagers la transmission de leur avis d'impôt. Cette plateforme fluidifie les échanges en permettant aux personnes physiques d'utiliser un compte partenaire de leur choix, le revenu fiscal de référence et le nombre de parts du foyer étant transmis automatiquement et de manière dématérialisée.

D'autres thématiques sont actuellement en cours d'expertise avec les villes de Paris, Lyon et Marseille, afin de simplifier la démarche d'obtention des cartes de stationnement résidentiel. II s'agit d'une véritable simplification pour les usagers, ainsi que d'une nouvelle valorisation des données partagées entre administrations publiques.

\section{Geneviève OLLIER, Comptable du Trésor}

Mesdames OLLIER et DUBOST, respectivement Comptable du Trésor et Directrice des finances de la Ville de Vincennes, ont apporté leur retour d'expérience quant au déploiement de la dématérialisation au sein de la ville de Vincennes. Comment les différentes directions s'étaient organisées afin de déployer ce nouveau processus? Quels en avaient été les gains concrets et quelles avaient été les difficultés apparues à l'occasion de ce déploiement.

1. À Vincennes, la démarche de dématérialisation a été instaurée dès 2005. L'ordonnateur et le comptable de Vincennes étaient désireux de s'engager dans une dynamique de progrès. Ils ont donc signé une charte de partenariat pour mettre en œuvre des prestations innovantes en vue de renforcer leur collaboration et d'accroître leur performance.

2. Le déploiement s'est fait progressivement et par étape, au regard de l'offre service:

- 2005 : mise en service, au niveau de la régie scolaire et périscolaire, du paiement en ligne par carte bancaire et du paiement par prélèvement automatique. Afin de réussir cette première étape, les régies ont été revues, regroupées et centralisées ;

- 2006 : mise en œuvre de la dématérialisation de la paie. Ce sont ainsi 36000 pages qui sont désormais économisées chaque année ;

- 2012 : adoption du protocole PES V2 pour la dématérialisation des pièces justificatives et leur transmission au comptable

- 2013 : réalisation de la «full dématérialisation» des pièces comptables, des mandats et des pièces justificatives ;

- 2015 : expérimentation des sommes à payer format XML vers Hélios et Chorus Pro. La ville a complété la mise en place du PES ASAP avec I'adoption du TIPI' ;

- 2016 : mise en service de la signature électronique. Ce dernier point a permis la constitution d'archives dématérialisées.

3. Les résultats recensés ont été, de manière générale, positifs. La dématérialisation a permis une fiabilisation accrue dans la qualité des comptes grâce à l'automatisation dans la passation des opérations comptables, une économie de papier, un gain de temps pour la mise en état d'examen des comptes auprès du juge financier, une facilitation de la recherche, un allègement des tâches, des délais de paiement raccourcis, une meilleure traçabilité et une transparence totale afin de permettre une évolution vers un travail qualitatif. Toutefois, on note un risque physique lié au travail sur écran entraînant une fatigue des agents.

\section{Anne-Marie DUBOST, Directrice des finances de la ville de Vincennes}

1. De façon pragmatique, il convient de réfléchir aux dispositifs qui prendront peu de temps à déployer et apporteront un gain immédiat aux utilisateurs. Par exemple, scanner les relevés d'identité des fournisseurs permet de revoir la saisie des tiers et d'en faciliter l'archivage. De la même manière, la modernisation des moyens de paiement apporte un confort pour les usagers, tout en permettant une meilleure efficacité du recouvrement.

2. La dématérialisation des factures de dépense est un volet demandant plus d'investissement en termes de temps, mais avec un retour sur le long terme non négligeable. À Vincennes, les services ont opté pour un scan manuel des factures au sein de leurs locaux. Ce choix a permis de ne pas engendrer de frais d'investissement dans un matériel spécifique. Afin d'inclure les factures en pièce jointe dans le logiciel CIVIL Net Finances, il a également fallu acquérir un certificat de signature électronique pour autoriser leur intégration. D'autre part, pour faciliter ce travail, aucun code référence n'a été imposé aux utilisateurs au début, l'objectif étant de récupérer les factures le plus rapidement possible.

Afin de créer un archivage électronique avec un classement homogène, il a fallu s'adapter aux services. Lorsque l'on impose un dispositif, il faut aussi pouvoir répondre à la diversité des services: penser à saisir les adresses emails des utilisateurs pour les relances par exemple ; créer des délégations permanentes, afin d'éviter des blocages de factures en cas d'absence; et bien entendu, communiquer au sein des services sur le sujet. 
À ce titre, la Ville a préféré ne pas communiquer en amont sur le projet de dématérialisation. II s'agissait de le consolider avant sa mise en production. La communication vers les services a donc réellement commencé lorsque le processus a été contrôlé en interne.

Des guides d'utilisateurs personnalisés ont été rédigés, dans un premier temps, pour les agents de la Direction des finances, puis pour les autres services, selon leurs actions. Des réunions pour l'ensemble des interlocuteurs de chaque service ont également été organisées.

3. En mettant en place la dématérialisation, la Ville a pu mettre en œuvre des reporting mensuels du suivi des factures par service, permettant un retour d'informations fluide et rapide sur l'acquittement de celles-ci. Les services ont également pu revoir la liste des pièces jointes transmises au comptable, avec pour objectif de réduire le délai de traitement des titres de recettes et donc de leur recouvrement.

Enfin, la dématérialisation sert également aujourd'hui à la transmission des budgets à la Préfecture. Les maquettes budgétaires étant réalisées à l'avance, il est possible de gagner du temps et d'éditer le document sous le format souhaité pour le transmettre, dès le budget voté, avec la délibération.

"Aujourd'hui, les collectivités n'ont plus besoin d'une décennie pour déployer la dématérialisation de bout en bout. Le partenariat avec la DGFiP a été essentiel pour déployer avec succès la dématérialisation».

\section{Pascale PRADELS,}

\section{Directrice financière et comptable de I'UGAP'}

Pionnier de la dématérialisation, I'UGAP a développé des solutions de dématérialisations originales. Pascale PRADELS a ainsi expliqué comment la dématérialisation contribue à sécuriser la chaîne comptable et à développer de nouveaux services.

1. Actuellement, I'UGAP traite plus de 1600 marchés à bons de commande et collabore avec plus de 600 fournisseurs (53\% de PME, 28 d'ETI $^{10}, 15 \%$ de grandes entreprises et $5 \%$ de ESAT ${ }^{11} /$ associations...). La question du délai de paiement est donc particulièrement sensible.

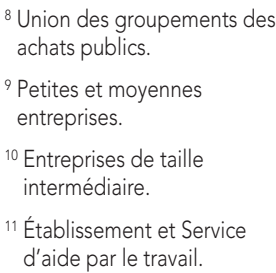

La dématérialisation et la mise en place d'un service facturier ont notamment favorisé la sécurité et le respect des délais de paiement. Grâce à la dématérialisation des flux et à la réception des factures en un point unique, les paiements sont assurés à 30 jours.
Les risques de non-réception des factures sont réduits et les modalités de contrôles sont maîtrisées, compte tenu des données intégrées dans le système d'information. Une trentaine de points de contrôle sont ainsi automatisés pour les factures dématérialisées (mentions obligatoires, cohérence des montants facturés avec le bon de commande...).

En outre, les gains de productivité ont permis de faire face, à effectif constant, à un quadruplement du nombre de factures traitées depuis 2006.

2. En tant qu'établissement public industriel et commercial, I'UGAP est tenu à une double obligation. À la différence des autres centrales d'achat public européennes, qui procèdent à la mise à disposition de cadres contractuels que leurs clients signent et exécutent eux-mêmes avec les fournisseurs retenus, I'UGAP revend à ses clients des fournitures ou services qu'elle a préalablement achetés.

Dans ce cadre, I'UGAP passe les procédures, signe les marchés, reçoit les commandes de ses clients. Elle les transmet ensuite aux fournisseurs sélectionnés, contrôle leurs factures, en assure le paiement, et enfin, facture ses clients publics. Tous les flux juridiques, commerciaux et financiers transitent donc par l'UGAP.

Le traitement de factures au format dématérialisé concerne ainsi tout à la fois les échanges avec les fournisseurs, titulaires de marchés et ceux avec les clients de l'ensemble de la sphère publique. Face à l'enjeu que cela représente (en 2016 : plus d'un million de factures fournisseurs, dont $76 \%$ ont été reçues de manière dématérialisée) il fallait maîtriser et rationnaliser les dépenses de l'établissement.

3. Sur le plan pratique, la réussite de la dématérialisation s'est faite par un projet commun entre l'ordonnateur et le comptable, tout en conservant l'idée que le projet doit avoir un sens dans la stratégie de l'établissement (être compétitif, proposer des offres performantes, maîtriser les coûts tout en développant son domaine d'activité) et être un travail collaboratif avec l'ensemble des directions de l'UGAP.

La dématérialisation a été réalisée en deux grandes étapes:

- 2006 à 2013 : réception dématérialisée des factures fournisseurs ;

- 2014 à 2016 : accès à la dématérialisation complète, en proposant aux fournisseurs d'adresser leurs factures sous format PDF signé via le portail d'échange de l'UGAP (SINOE).

Une réflexion a dû être menée pour accompagner cette démarche par le biais d'un cadre technique, mais aussi d'une organisation et d'un processus 
de traitement des factures. Une équipe a été entièrement dédiée à ce projet.

«Il faut accepter l'idée que le retour sur investissement ne soit pas immédiat. (...). L'accompagnement des équipes et le dialogue social sont essentiels pour réussir un projet de dématérialisation ».

Mais il faut également penser à accompagner les équipes, puisque des inquiétudes peuvent survenir face au changement de métier. En effet, les salariés effectuant la saisie des factures ont vu leurs missions évoluer et c'est cette évolution qu'il faut accompagner, afin de ne pas susciter de résistances. D'où la nécessité de s'assurer de la bonne diffusion des informations auprès, notamment, des instances représentatives du personnel.

Enfin, même si la dématérialisation apporte beaucoup d'avantages, elle peut générer une fatigue liée au travail quotidien sur écran pour les salariés.

Parmi les gains obtenus, il faut également insister sur la sécurisation et fiabilisation des échanges et l'accès en temps réel aux statuts des factures et à leur traitement par les clients.

4. Parallèlement aux travaux liés à la dématérialisation des factures, I'UGAP a développé d'autres outils visant à la dématérialisation des procédures d'achat, à la gestion des contrats (accords-cadres et marchés) et des commandes adressées à ses fournisseurs ou reçues de ses clients.

L'outil interne, baptisé «ENOMA » (Établissons Nos Marchés), permet de garantir le respect de la formalisation des documents de consultation et d'opérer le suivi « pas à pas » de chaque étape de la procédure. Ces procédures sont publiées et gérées au travers du profil acheteur PLACE élaboré par l'État. Cette plateforme permet les échanges avec les candidats, la réception de leurs offres puis la notification des contrats aux attributaires.

Les contrats dématérialisés sont intégrés dans un système de gestion documentaire (E-Magin) qui permet à l'ensemble des collaborateurs de l'établissement de disposer d'un accès à ces supports sur la base de profils et d'habilitations spécifiques.

En phase d'exécution des marchés, les commandes sont adressées aux fournisseurs de manière dématérialisée, soit par échanges de données informatisées (EDI) de système à système, soit déposées, au format PDF, sur le portail fournisseurs dénommé «Sinoé » (SImplifions Nos Echanges). Ce portail permet également, au niveau de chaque commande, quel que soit son mode de transmission, des échanges dématérialisés avec les titulaires de marché ainsi qu'une centralisation, un archivage et un partage de toutes les informations avec l'ensemble des métiers et fonctions de l'établissement.

L'UGAP a par ailleurs développé, depuis 2009, un site de visualisation de son offre et de commande en ligne ugap.fr qui enregistre désormais plus de $43 \%$ des lignes de commandes traitées par l'établissement. Ce basculement vers la dématérialisation de la commande client a ainsi permis de concentrer les ressources de l'UGAP sur la relation de proximité avec les donneurs d'ordres publics.

\section{Les rendez-vous de la transformation de la fonction finance au sein du bloc local}

La constitution de nouvelles collectivités de grande taille (régions, métropoles, établissements publics de coopération intercommunale), l'évolution des modes de financement des investissements, la généralisation progressive du chantier de la dématérialisation, l'ouverture des données à caractère budgétaire et l'officialisation du lancement de la phase expérimentale de la certification des comptes du bloc local constituent les évènements récents les plus significatifs d'une transformation en profondeur de la fonction finance au sein des collectivités territoriales.

Dans ce contexte, la Revue Gestion \& Finances Publiques et le Forum pour la Gestion des Villes et des Collectivités Territoriales, organisent : les Rendez-vous de la Transformation de la Fonction Finance (RTFF). Les thématiques abordées sont les suivantes :

1. De la dématérialisation à la digitalisation de la fonction Finance : enjeux et opportunités pour le bloc local

2. La mobilisation des équipes dans un projet de transformation et de digitalisation de la fonction finance
3. Comment faire de votre système d'information un levier de performances dans la réalisation de votre projet de dématérialisation?

4. Mutualisation de la fonction finance et centre de services partagés

5. La gestion dynamique du patrimoine : comment rentabiliser l'actif de votre collectivité?

6. Audit des projets et investissements (article 108 de la NOTRe) : comment arbitrer et piloter un portefeuille d'investissements (CAPEX) ?

7. Certification des comptes des collectivités candidates : un $1^{\text {er }}$ retour d'expériences

8. Demain, la fonction Finance en mode disruptif : cybersécurité, datamining, algorithmes, blockchain

La Revue Gestion \& Finances Publiques publiera la synthèse de chacun des RTFF.

Renseignements sur : www.forum-gv.com 